\title{
Tabagismo entre gestantes atendidas em maternidade filantrópica do Município de São Paulo\#
} Smoking among pregnant women attending philanthropic maternity in São Paulo (Brazil)

\author{
Natália Machado Costa Lopes* \\ Maria Alice Tsunechiro** \\ Alfredo Almeida Pina-Oliveira*** \\ Anna Maria Chiesa****
}

\begin{abstract}
RESUMO
O consumo do tabaco continua sendo um dos problemas de saúde, sobretudo no período gestacional. Os objetivos do estudo foram verificar o comportamento dos fumantes na gestação no que se refere: à prevalência de tabagismo, à caracterização da história tabagística da gestante, aos níveis de dependência à nicotina e à relação entre o número de cigarros consumidos e o peso do recém-nascido. Foram entrevistadas 273 puérperas, entre novembro de 2012 e janeiro de 2013, em uma maternidade filantrópica de São Paulo. Os resultados revelaram prevalência de $11 \%$ de gestantes fumantes, a maioria com grau leve de dependência à nicotina. A média da idade de início do consumo de cigarro foi 15,6 anos. Os recém-nascidos de mulheres fumantes apresentaram peso de nascimento estatisticamente menor quando comparados com filhos de mulheres não fumantes. A abordagem do tabagismo no pré-natal é insuficiente, a maioria é indagada sobre o consumo do cigarro, porém, a orientação quando feita, é em sua maioria às mulheres que fumam. O conhecimento das mulheres referente ao tabagismo e à saúde do bebê é baixo, os problemas respiratórios são os mais lembrados; 35,9\% das mulheres não souberam informar nenhuma patologia associada ao cigarro e ao bebê. Quanto aos prejuízos à saúde materna, o câncer foi o mais lembrado. Observou-se ser necessário intensificar a abordagem sobre o tabagismo no pré-natal, para alertar sobre os riscos e malefícios do cigarro à mãe e ao bebê, pois há ainda muitas mulheres que desconhecem as informações básicas sobre o assunto.
\end{abstract}

Palavras-chave: Tabagismo - efeitos adversos. Tabagismo - epidemiologia. Gravidez. Cuidado.

\section{ABSTRACT}

The study aimed to verify the behavior of smoking during pregnancy in relation to: the prevalence of smoking, the characterization of the smoking history of the mother, the levels of nicotine dependence and the relationship between the number of cigarettes smoked and the birth weight of newborns. There were interviewed 273 mothers between November 2012 and January 2013, at a philanthropic maternity hospital in Sao Paulo. The results revealed a prevalence of $11 \%$ of pregnant smokers; most of them had mild nicotine dependence. The average age of onset of cigarette consumption was 15,6 years. The birth weight from newborns of smoking mothers was statistically lower in comparison to the birth weight from newborns of non-smoking women. The approach of prenatal smoking is insufficient, in which the majority of women are asked about the consumption of cigarettes, but the guidance is performed mostly for women who smoke. Thus, women's knowledge about smoking and newborn health is low, respiratory problems are the most remembered by them; however, $35.9 \%$ of women were unable to name any pathology associated with cigarette and the newborn. About the harms to maternal health, cancer was the most remembered. It was observed that it is necessary to intensify the approach of smoking at prenatal period, warning pregnant women about the risks and harms caused by cigarette for both the mother and the newborn, as there are still a lot of women who do not know basic information about the subject.

Keywords: Smoking - adverse effects. Smoking - epidemiology. Pregnancy. PrenatalCare.

DOI: 10.15343/0104-7809.20153901102112

\# Artigo derivado do Trabalho de Conclusão de Curso "Comportamento do fumar no período gestacional" apresentado ao Curso de Especialização em Enfermagem Obstétrica, Centro Universitário São Camilo, 2013.

* Centro Universitário São Camilo. São Paulo, Brasil.

** Universidade de São Paulo, São Paulo, Brasil.

*** Faculdade de Medicina da Universidade de São Paulo, Brasil.

**** Universidade de São Paulo. São Paulo, Brasil.

Os autores declaram não haver conflitos de interesse. 


\section{INTRODUÇÃO}

Ao longo das últimas décadas, muito vem se discutindo sobre o tabagismo. Dados da Organização Mundial de Saúde (OMS) revelam que o consumo do tabaco continua sendo uma das principais causas de mortes no mundo, que podem ser evitáveis. Mais de seis milhões de pessoas morrem a cada ano, e o impacto na economia global é de cerca de centenas de bilhões de dólares. A longo prazo, se nenhuma medida efetiva for tomada urgentemente, a expectativa será que o uso do tabaco possa matar mais de um bilhão de pessoas somente neste século .

O tabagismo é responsável por cerca de cinquenta doenças, a maioria delas relacionadas ao sistema cardiopulmonar, como infarto agudo do miocárdio, bronquite crônica e enfisema pulmonar. O tabaco é responsável por 90\% dos casos de câncer de pulmão e, em 2010, houve mais de 21.000 mortes por causa do câncer do aparelho respiratório. O uso do tabaco também é responsável por 30\% das mortes decorrentes de outros tipos de câncer (boca, laringe, faringe, esôfago, estômago, pâncreas, fígado, rim, bexiga, colo de útero e leucemia) ${ }^{2}$. De acordo com dados do Ministério da Saúde, a estimativa no Brasil para o ano de 2012 foi de mais de 17.000 casos novos de câncer de pulmão em homens e mais de 10.000 em mulheres. Mais de 200.000 mortes por ano são causadas pelo tabaco no Brasil $^{3}$.

\section{Tabagismo e gestação}

No Brasil, de acordo com o relatório da OMS referente ao ano de 2010 sobre a epidemia do tabaco no mundo, a prevalência de fumantes do gênero feminino foi de $13 \%{ }^{1}$.

Além das diversas doenças provocadas pelo tabagismo, na população feminina a preocupação maior é com as mulheres com possibilidade de gestação. Sabe-se desde há muito tempo dos danos causados pelo consumo do tabaco durante a gravidez, sobretudo no desenvolvimento da placenta.
Em estudo realizado em um município do Rio Grande do Sul, a prevalência de tabagismo materno durante a gestação foi de $23 \%$, um número que vem diminuindo se comparado com outras pesquisas realizadas desde 1982, quando esse número era de 35,6\%. Os autores destacam que, apesar da redução, a prevalência de gestantes fumantes ainda é alta se forem comparados números de países industrializados (10\% -13\%) e algumas cidades sul-americanas $(10,3 \%-18,3 \%)^{4}$.

O tabagismo causa diminuição da pressão de oxigênio materno, comprometendo a perfusão adequada da placenta e a absorção de nutrientes para o feto ${ }^{5}$. Assim, o desenvolvimento do feto é prejudicado. O consumo de drogas e as atividades contraindicadas para o período gravídico afetam o crescimento intrauterino, associando a diminuição do peso fetal em 135 a 300g6. Um estudo feito em Porto Alegre (RS), revelou que, no grupo de mulheres fumantes, o número de recém-nascidos pequenos para idade gestacional (PIGs) foi expressivamente maior ${ }^{5}$.

As mulheres iniciam o comportamento de fumar como forma de se inserir socialmente, seja em grupos de amigos ou mesmo na família, a fim de se aproximarem mais das pessoas e de reduzir a ansiedade. As mulheres veem o cigarro como fonte de satisfação e companhia. Mas, quando ele é associado à gestação, outros sentimentos são manifestados, como medo das consequências, culpa, raiva e agonia. Os sintomas da abstinência e as sensações prazerosas conflitam na decisão de parar de fumar, levando as mulheres a acharem que é um vício impossível de ser abandonado ${ }^{7}$.

No estudo realizado em Porto Alegre (RS), foi verificado que $99 \%$ das mulheres sabiam dos malefícios do uso do tabaco, tanto durante a gestação como após o parto, mas mesmo assim elas não interromperam o uso, demonstrando o que chamaram de negligência consciente por parte das mulheres ${ }^{5}$.

Mais do que o problema econômico que o hábito de fumar acarreta na economia de um 
país, aos gastos por possíveis intercorrências causadas pelo tabagismo durante a gestação, são acrescentados os gastos advindos dos cuidados específicos dispensados, tanto às mulheres como aos neonatos e à criança no primeiro ano de vida, sobretudo ligados a problemas respiratórios. Assim, a consulta pré-natal é uma boa oportunidade de esclarecer e alertar sobre os malefícios do fumar na gestação, além de amparar a gestante em seus medos, dúvidas e angústias do momento por que passam ${ }^{8}$. Trata-se de um aspecto que pode ampliar a clínica do Pré-Natal na perspectiva da integralidade em saúde.

Justifica-se realizar este estudo considerando a dimensão que a problemática do fumar na gestação acarreta não só à saúde da mulher e do recém-nascido, como também para o sistema de saúde e econômico do País. Assim, ao identificar a prevalência das gestantes fumantes, a história tabagística e o nível de dependência à nicotina, serão possíveis traçar um perfil sobre essa população e verificar se as campanhas de combate ao fumo e as consultas pré-natal estão sendo eficazes.

Os objetivos deste estudo foram: verificar a prevalência do comportamento de fumar entre gestantes, caracterizar a história tabagística da gestante, identificar os níveis de dependência à nicotina e verificar a relação entre o número de cigarros e o peso do recém-nascido.

\section{METODOLOGIA}

Trata-se de um estudo transversal sobre o comportamento do fumar no período gestacional.

A pesquisa foi desenvolvida no Amparo Maternal, maternidade filantrópica da cidade de São Paulo, que presta atendimento obstétrico de baixo risco exclusivamente pelo Sistema Único de Saúde (SUS) a mulheres provenientes de todas as regiões da capital e demais municípios da Grande São Paulo. Em 2011, o número de partos na maternidade foi de, aproximadamente, 500 por mês.
A população do estudo constituiu-se de mulheres no pós-parto mediato internadas na unidade de puerpério (normal e cirúrgico) da referida maternidade.

Os critérios de inclusão das mulheres no estudo foram:

- Idade entre 18 e 35 anos;

- Gestação a termo (idade gestacional $\geq 37$ semanas) com feto vivo;

- Ter frequentado serviço de saúde para seguimento pré-natal;

- Ausência de gestação múltipla e de patologias na gestação, como hipertensão arterial, doença hipertensiva específica da gravidez, Diabetes mellitus, entre outras. A elegibilidade das participantes foi definida com base nesses critérios de inclusão e consulta aos prontuários. Os dados foram obtidos por meio de entrevista individual realizada por duas pesquisadoras da equipe, no período compreendido entre novembro de 2012 e janeiro de 2013.

As mulheres foram convidadas a participar do estudo, após os esclarecimentos sobre a pesquisa, sendo informadas de que o estudo não oferecia risco imediato, porém, que existia a possibilidade de um risco subjetivo, pois algumas perguntas remetiam à autoavaliação de comportamento em saúde. Assim, o possível risco referia-se à lembrança de sua história de vida e às consequentes repercussões do fumo em sua saúde e na do bebê, mas, que a pesquisa traria benefícios à população estudada, pois resultaria em melhor atendimento às mulheres grávidas e ao filho esperado.

Após a concordância em participar do estudo, foi realizada a entrevista em local privativo e reservado da unidade de puerpério da maternidade sendo solicitada a assinatura do TCLE Termo de Consentimento Livre e Esclarecido.

$\mathrm{O}$ instrumento para coleta de dados foi um formulário com três partes: I - dados da puérpera e do recém-nascido; II - história tabagística; III - avaliação do grau de dependência de nicotina - Teste de Fagerström (respondido apenas pelas fumantes). 
O Teste de Fagerström é usado para estabelecer o grau de dependência da nicotina nos fumantes com o propósito de direcionar o tratamento mais adequado para cada pessoa, sendo composto de seis questões, das quais duas têm quatro alternativas com graus de intensidade variando de 0 a 3 pontos, e quatro possuem duas alternativas, ou 0 ou 1 . O somatório dos pontos de cada questão permite avaliar o grau de dependência: $0-2$ pontos = Muito baixo, $3-4$ pontos $=$ Baixo, 5 pontos $=$ Médio, $6-7$ pontos = Elevado e $8-10$ pontos = Muito elevado. Sintomas da síndrome de abstinência são mais prováveis de ocorrer quando o somatório dos pontos é igual ou superior a 6 .

Os dados coletados foram armazenados em planilha com digitação em dupla entrada, utilizando o programa Excel Microsoft 2010.

Para a caracterização da amostra, os dados foram distribuídos, conforme mulheres não fumantes, ex-fumantes e fumantes. As variáveis socioeconômicas e obstétricas foram avaliadas em suas frequências absolutas e relativas. Os pesos dos recém-nascidos foram analisados por meio de estatística descritiva com o cálculo das medidas de tendência central - mediana, média e desvio-padrão.

A relação entre os valores médios do peso do recém-nascido e o número de cigarros fumados por dia foi analisada pelo Teste de Kruskal-Walis e para a comparação entre as médias dos pesos dos recém-nascidos de mães não fumantes e fumantes foi utilizado o teste de Wilcoxon.

Para verificar a associação do grau de dependência de nicotina e a quantidade de cigarros fumados por dia, foi utilizado o Teste Exato de Fisher.

O nível de significância adotado para este estudo foi de $5 \%$, portanto, os valores de $p$ menores que 0,05 ( $p$-valor 0,05 ) foram apontados como estatisticamente significantes.

O projeto foi aprovado pelo Comitê de Ética em Pesquisa do Centro Universitário São Camilo (Parecer no 129.150) em outubro de 2012.
A coleta de dados foi feita após a autorização do responsável pela instituição coparticipante.

Todas as mulheres entrevistadas assinaram e receberam uma cópia do TCLE. Para aquelas que mostraram desconhecimento ou conhecimento insuficiente e/ou inadequado foram feitas orientações pertinentes aos prejuízos do cigarro à saúde do bebê e das pessoas que fumam ou convivem com fumantes.

\section{RESULTADOS}

No período estudado, foram entrevistadas 273 mulheres no pós-parto internadas nas unidades de puerpério e que compuseram a amostra do estudo.

Com relação às características das entrevistadas, praticamente a metade delas foi constituída de jovens entre 18 e 24 anos (49,5\%); a maioria $(81,0 \%)$ com parceiro (por casamento ou união consensual); 73,3\% com escolaridade superior a 9 anos de estudo; 48,0\% primigesta e $88,3 \%$ com seis ou mais consultas de pré-natal.

\section{Prevalência, níveis de dependência à nico- tina e a relação do tabagismo e o peso do recém-nascido.}

O consumo de cigarro na gestação foi observado em $11,0 \%$ das mulheres do presente estudo, 19,8\% como ex-fumantes e pouco mais de dois terços $(69,2 \%)$ nunca fumaram. As ex-fumantes foram mulheres que alegaram que deixaram de fumar, pouco antes da gravidez ou há alguns anos.

A pontuação do Teste de Fagerström obtida pelas mulheres fumantes mostrou que dois terços $(66,7 \%)$ apresentaram grau de dependência à nicotina Muito baixo ou Baixo, e pouco mais de um quarto $(26,6 \%)$ obteve altos índices de dependência.

Nos dados da Tabela 1, pode-se verificar que há diferença estatisticamente significativa, $p=0,040$, e quanto maior o número de cigarros consumidos maior será o nível de dependência da nicotina. 
Tabela 1. Grau de dependência da nicotina, conforme a quantidade de cigarros consumidos por dia pelas mulheres fumantes. São Paulo, 2013.

\begin{tabular}{|c|c|c|c|c|c|c|c|c|}
\hline \multirow{3}{*}{$\begin{array}{l}\text { Grau de dependência } \\
\text { da nicotina }\end{array}$} & \multicolumn{6}{|c|}{ Quantidade de cigarros } & & \\
\hline & \multicolumn{2}{|c|}{$1-5$} & \multicolumn{2}{|c|}{$6-10$} & \multicolumn{2}{|c|}{$\geq 11^{*}$} & \multicolumn{2}{|c|}{ Total } \\
\hline & $\mathrm{n}$ & $\%$ & $\mathrm{n}$ & $\%$ & $\mathrm{n}$ & $\%$ & $\mathrm{n}$ & $\%$ \\
\hline Muito baixo & 3 & 20,0 & 7 & 46,7 & 5 & 33,3 & 15 & 100,0 \\
\hline Baixo & 1 & 20,0 & 1 & 20,0 & 3 & 60,0 & 5 & 100,0 \\
\hline Médio & - & - & 2 & 100,0 & - & - & 2 & 100,0 \\
\hline Elevado & - & - & - & - & 7 & 100,0 & 7 & 100,0 \\
\hline Muito elevado & - & - & - & - & 1 & 100,0 & 1 & 100,0 \\
\hline
\end{tabular}

*Número máximo de cigarros $=40$

Valor-p $=$ 0,040 (teste exato de Fisher)

Observa-se nos dados da Tabela 2 que os valores mostram a associação estatisticamente significativa $(p=0,014)$ do comportamento do fumar com o peso do recém-nascido. A média dos pesos dos recém-nascidos das mulheres que não fumaram na gestação é 210 gramas (g) maior do que a média do peso dos filhos das mulheres fumantes.

Tabela 2. Mediana, Média, desvio padrão (DP) Valor Mínimo (Vmin) e Valor Máximo (Vmax) do peso (g) do recém-nascido de mãe não fumante e fumante na gestação atual. São Paulo, 2013.

\begin{tabular}{lcccccc}
\hline \multirow{2}{*}{ Comportamento do fumar } & \multicolumn{7}{c}{ Peso (g) do recém-nascido } \\
\cline { 2 - 7 } & Mediana & Média & DP & Mínimo & Máximo & Total \\
\hline Não Fumante & 3225 & 3263 & 410 & 2350 & 4325 & 243 \\
Fumante & 3100 & 3053 & 430 & 2325 & 4105 & 30 \\
\hline
\end{tabular}

Valor-p: 0,014 (teste Wilcoxon)

Tabela 3. Mediana, Média, Valor Mínimo (Vmin) e Valor Máximo (Vmax) do peso do recém-nascido em função da quantidade de cigarros fumados por dia pela mãe. São Paulo, 2013.

\begin{tabular}{lcccccc}
\hline \multirow{2}{*}{ Quantidade de cigarros } & \multicolumn{7}{c}{ Peso (g) do recém-nascido } \\
\cline { 2 - 7 } & $\mathrm{n}$ & Mediana & Média & DP & VMin & Vmax \\
\hline $0^{*}$ & 243 & 3225 & 3262 & 410 & 2350 & 4325 \\
$1-5$ & 4 & 2655 & 2861 & 653 & 2325 & 3810 \\
$6-10$ & 10 & 3112 & 3059 & 427 & 2385 & 3665 \\
11 e mais & 16 & 3128 & 3111 & 387 & 2435 & 4105 \\
\hline
\end{tabular}

* Não-fumantes e ex-fumantes

Valor-p = 0,080 (teste de Kruskal-Wallis)

Os valores apresentados nos dados da Tabela 3 mostram que não há associação significativa entre o número de cigarros fumados por dia e as médias dos pesos dos recém-nascidos. 


\section{Conhecimento e orientação sobre o tabagismo na gestação e história tabagistica das mulhe- res fumantes e ex-fumantes}

Uma proporção relativamente alta de muIheres $(89,4 \%)$ afirmou que durante a consulta pré-natal foram indagadas sobre o consumo do cigarro. Quanto à orientação sobre o fumar na gestação, a proporção maior foi observada nas mulheres fumantes $(60,0 \%)$, e pouco mais de um terço $(38,9 \%)$ das ex-fumantes e menos de um quarto $(22,8 \%)$ das não fumantes receberam orientação sobre o tabagismo na gestação.

O câncer foi o problema mais lembrado pelas mulheres $(56,2 \%)$, como repercussão para a saúde da pessoa que fuma e, embora em pequena proporção, 4,7\% das mulheres não souberam informar, pelo menos, uma doença causada pelo cigarro. Como repercussão à saúde do bebê, os problemas respiratórios (44,3\%) foram os mais lembrados pelas entrevistadas. Vale ressaltar que $35,9 \%$ das puérperas não souberam descrever, pelo menos, um prejuízo que o cigarro traz ao bebê.

O início do consumo de cigarro ocorreu na adolescência, em torno de 15 a 16 anos de idade, a mais precoce aos 10 anos e uma delas aos 28 anos (Tabela 4).

Tabela 4. Média, desvio padrão (DP) Valor Mínimo (Vmin) e Valor Máximo (Vmax) da idade de início do consumo de cigarro de mulheres ex-fumantes e fumantes. São Paulo, 2013.

\begin{tabular}{lccccc}
\hline \multirow{2}{*}{ Comportamento do fumar } & \multicolumn{4}{c}{ Idade (anos) início do consumo de cigarro } \\
\cline { 2 - 6 } & Média & DP & Mínimo & Máximo & Total \\
\hline Ex-Fumante e Fumante & 15,6 & 2,8 & 10 & 28 & 84 \\
Ex-Fumante & 15,5 & 2,3 & 11 & 23 & 54 \\
Fumante & 15,6 & 2,8 & 10 & 28 & 30 \\
\hline
\end{tabular}

As mulheres informaram que o início do comportamento do fumar ocorreu por curiosidade própria $(42,7 \%)$, seguida por influência de colegas e amigos de escola $(37,1 \%)$, entre outros. Dentre as razões do fumar, as principais apontadas pelas mulheres foram a sensação de relaxamento e redução da tensão (59,5\%) e a dependência comportamental relacionada ao tabaco (44,0\%).

\section{Razões para cessar o fumar e motivos para recaída}

Quanto ao parar de fumar, a maioria das mulheres (96,7\%) expressou ter esse desejo, e uma respondeu que não sabia se queria parar de fumar. A gravidez foi a razão para cessar o fumar mais apontada pelas mulheres (54,9\%); seguida da vontade de cessar o fumar em $49,3 \%$ das respostas, dos sintomas físicos, como cansaço e problemas respiratórios (19,7\%) e a influência do cônjuge (11,3\%).

Das mulheres fumantes, $80,0 \%$ alegaram que diminuíram o número de cigarros fumados por dia e apenas 10,0\% conseguiram parar de fumar na gestação, sobretudo no final, dada a proximidade do parto.

As mulheres com história de recaída mencionaram que o convívio com fumantes $(52,6 \%)$ foi o principal motivo que as levou a voltarem a fumar, seguido de condições alusivas aos aspectos emocionais e de enfrentamento de adversidades (dependência psicológica associada ao consumo do tabaco).

\section{DISCUSSÃo}

As mulheres grávidas, objeto desta pesquisa, tinham idade entre 18 e 35 anos, quase metade (49,5\%) encontrava-se na faixa entre 18 e 24 anos. A maioria era de não fumante, tinha parceiro fixo e escolaridade superior a 9 anos de estudo, 48\% delas estavam na primeira gestação e frequentaram seis ou mais consultas pré-natais.

Os resultados do presente estudo mostraram que a questão do tabagismo necessita ainda de atenção, como problema de saúde pública e merece destaque na saúde da gestante e do recém-nascido. 
A prevalência do tabagismo materno de $11 \%$ representa um declínio significativo se for comparada com o estudo realizado em 2001 no mesmo serviço, cujos achados evidenciaram $25,6 \%$ de mulheres que fumaram no período gestacional ${ }^{9}$, o que sugere que as ações governamentais implementadas na última década para o controle do tabagismo vêm alcançando resultados efetivos.

Estudos brasileiros com dados dos últimos 10 anos apontam prevalências que variam de $5,5 \%$ a $23,3 \%$. Um estudo transversal realizado em uma maternidade-escola pública do Rio de Janeiro (RJ) sobre consumo de álcool e cigarro na gestação, com uma amostra de 433 puérperas atendidas, entre 1999 e 2006, mostrou que $5,5 \%$ eram fumantes e $7,4 \%$ consumiram álcool e que o uso do cigarro foi associado ao do álcool ${ }^{11}$.

Por sua vez, em pesquisa realizada com 486 gestantes em clínicas de pré-natal de quatro centros de saúde na cidade do Rio de Janeiro (RJ), entre abril de 2003 e fevereiro de 2004, $21,1 \%$ eram mulheres fumantes nos estágios iniciais da gravidez ${ }^{12}$.

Na cidade de Rio Grande (RS), verificou-se uma proporção de $23,3 \%$ de mulheres que referiram fumar durante toda a gestação em uma amostra de mães e recém-nascidos vivos em $2007^{4}$. Em outra pesquisa realizada em Porto Alegre (RS), com uma amostra de 267 puérperas, a prevalência de fumantes foi de 19,1\% ${ }^{13}$.

No estudo atual, das mulheres fumantes, a maioria $(66,7 \%)$ apresentou grau de dependência à nicotina considerado leve (Muito baixo e Baixo), semelhante ao verificado em outros estudos $^{10,12}$. Observou-se associação estatisticamente significativa entre o grau de dependência e o número de cigarros fumados por dia. Como esperado, as mulheres que obtiveram pontuação maior no Teste de Fagerström foram as que consumiam maior número de cigarros por dia, confirmando o achado em estudo semelhante ${ }^{10}$, que apontou grau de elevado ou muito elevado nas mulheres que consumiam mais de dez cigarros diários.
O grau de dependência da nicotina, obtido por meio do Teste de Fagerström, norteia o meIhor tratamento a ser adotado com o fumante. A pessoa que obtiver 6 pontos ou mais terá mais dificuldade ao tentar parar de fumar ${ }^{14}$.

O grau de dependência obtido pelo Teste de Fagerström contribui para a definição do cuidado ao fumante. O número de cigarros fumados por dia e o tempo após acordar e acender o primeiro cigarro são perguntas sensíveis à presença de síndrome de abstinência durante a cessação do fumar. Ademais, entre as mulheres que param de fumar na gestação, dois terços apresentam recaída após a gestação, indicando a necessidade de abordagem e o acompanhamento profissional dessa população ${ }^{15}$.

Quando questionadas sobre os malefícios do cigarro na saúde do recém-nascido, um terço das mulheres não soube informar, pelo menos, uma patologia que pudesse ser associada ao uso do cigarro na gestação. Entretanto, os resultados indicaram que o problema respiratório foi o mais associado aos malefícios do fumar na gestação. Em estudo realizado com 255 parturientes ${ }^{16}$, a maioria das mulheres $(94,1 \%)$, quando perguntadas sobre o fumo e a saúde da criança, citou problemas respiratórios, seguidos de problemas de malformação (59,2\%). Mas, quando indagadas dos prejuízos do cigarro à própria saúde, mais de 90,0\% das puérperas souberam informar pelo menos um.

Em estudo para avaliar a efetividade dos avisos sobre os malefícios do fumo presentes em rótulos de cigarro e também o conhecimento dos fumantes referente ao assunto, foi possível observar que a informação que os fumantes tinham era limitada, e que o câncer de pulmão foi o risco associado mais lembrado. Contudo, considera-se que as advertências presentes nos rótulos são formas importantes de levar informação, como estratégia populacional ${ }^{17}$.

Com relação ao desenvolvimento do feto, particularmente ao ganho ponderal, notou-se diferença de $210 \mathrm{~g}$ entre as médias de peso de nascimento de filhos de mulheres fumantes e não fumantes. Quando comparadas as médias do peso ao nascer de bebês de mães fumantes 
e não fumantes foi observada diferença estatisticamente significativa. Essa associação não foi observada quando foi considerado o número de cigarros fumados por dia ou não pelas mulheres. No entanto, observou-se que, entre as fumantes, o aumento do número de cigarros fumados foi acompanhado pelo aumento da média do peso do recém-nascido das mães fumantes, o que não corresponde aos efeitos atribuídos ao tabaco em relação ao peso do concepto, indicando a influência de outros fatores.

O fumar durante a gestação, como fator de redução do peso ao nascer, é um achado frequente na literatura. Dados similares foram observados em outros estudos que mostraram diferença entre 190 gramas e 223 gramas dos pesos dos recém-nascidos filhos de mães fumantes e de não fumantes ${ }^{4,16}$. Em estudo com 758 puérperas para analisar as repercussões do tabagismo na gestação e no recém-nascido ${ }^{18}$, os bebês de mulheres fumantes ativas ou passivas apresentaram menor peso de nascimento em comparação com os bebês de mulheres não fumantes e as que não tinham contato com a fumaça do cigarro.

O baixo peso ao nascer pode ser associado ao hábito de fumar da mãe que prejudica o desenvolvimento da placenta e do feto. As alterações ocorrem no metabolismo e oxigenação da placenta e, secundariamente, no próprio metabolismo do feto devido, sobretudo, a dois componentes do cigarro: a nicotina e o monóxido de carbono $(\mathrm{CO})^{19}$.

As repercussões negativas do cigarro na gestação como potencial causador de complicações fetais e maternas, bem como algo que pode levar à morte, fazem parte da representação de gestantes tabagistas ${ }^{7}$.

Vários estudos apontam a gravidez como um dos momentos mais propícios para a cessação do tabagismo ${ }^{12,20,21,22}$. Dessa forma, os estudos mostram que o sucesso na cessação do tabagismo é maior quando é direcionado às gestantes.

Trabalho realizado com uma amostra de 426 sujeitos $^{23}$, os fumantes só consideram parar de fumar, quando apresentam problemas de saúde relacionados ao cigarro. Este estudo mostrou também que a influência de pessoas próximas e a vontade própria e determinação de parar de fumar são os principais motivos que fazem com que a pessoa pare com o tabagismo.

Este trabalho confirmou os achados já demonstrados por outros estudos brasileiros sobre o início do hábito de fumar na adolescência, como a idade média de 15,4 anos ${ }^{9}$, a de 15 $\operatorname{anos}^{12}$, a idade entre 10 e $18 \operatorname{anos}^{13}$, entre outros. Nesse sentido, o tabagismo configura um problema que deve ser abordado no contexto pediátrico e hebiátrico, pois a experimentação precoce do tabaco em suas diferentes formas predispõe o indivíduo à maior probabilidade de desenvolver a dependência em idades posteriores. Assim, para se reduzir o tabagismo na gestação, as adolescentes devem constituir o alvo prioritário nos programas de promoção e educação em saúde.

A iniciação do tabagismo está associada à influência de colegas e familiares que fumam ${ }^{20}$. A resposta para o início desse comportamento na adolescência pode estar relacionada ao desenvolvimento da personalidade nessa fase, à necessidade de aceitação e à afirmação perante o grupo.

Os motivos que levam as mulheres a fumar são dependência física (vício), redução da tensão, manuseio do cigarro, controle de peso e associação estreita (conexão emocional), além do tabagismo social ${ }^{15}$. Nesse sentido, os motivos que levam ao uso do cigarro parecem estar ancorados no significado social que essa prática proporciona: aproxima as pessoas, favorece vínculos e acalma a ansiedade ${ }^{7}$.

Os resultados deste estudo indicaram que a abordagem do tabagismo pelos profissionais de saúde, especificamente na gestação, em geral, restringe-se a quem é fumante. Mesmo assim, as informações são limitadas e superficiais. $\mathrm{Na}$ última Pesquisa Nacional por Amostra de Domicílio - PNAD, do ano de 2008, na Pesquisa Especial de Tabagismo, que traçou o panorama atual do tabagismo no Brasil ${ }^{24}$, ficou evidente a falta de abordagem dos fumantes pelos profissionais de saúde. Apenas 57,1\% dos fumantes brasileiros haviam sido advertidos a parar de fumar nos últimos 12 meses. 
Não obstante, na literatura científica há sempre a menção da necessidade da abordagem e orientação sobre o tabagismo, sobretudo nos serviços de pré-natal, visto que a gravidez é uma condição que leva sempre a mulher procurar ajuda profissional, e essa oportunidade não deve ser desperdiçada.

Assim, consideramos que os profissionais de saúde, em especial, os obstetras, pediatras, enfermeiros e agentes de saúde são "a base do sucesso dos programas de abordagem do fumo na gravidez e primeira infância"25.

A vontade de parar de fumar é predominante entre as participantes deste estudo. Contudo, as mulheres entrevistadas apontaram dificuldades na cessação, apesar dos efeitos nocivos do tabagismo. Por essa razão, os profissionais de saúde devem reconhecer a importância da avaliação da dependência da nicotina e da motivação da mulher a fim de realizar aconselhamento personalizado, realista, empático, contextualizado e compartilhado ${ }^{26,27}$.

Modelos de aconselhamento empregados na adoção de hábitos saudáveis devem ajudar os profissionais de saúde na sistematização desse processo. O modelo PANPA consiste em uma intervenção breve que compreende cinco ações, a saber: perguntar, aconselhar, negociar, preparar e acompanhar. Tal método visa a oferecer o suporte logístico para o emprego de outros modelos e teorias que auxiliam os indivíduos no processo de mudança, em especial, no pensar, sentir e agir relacionados ao fumar ${ }^{26,27}$.

Cabe destacar que perguntar sobre alguns fatores pode aumentar a taxa de sucesso na cessação do tabagismo dos programas de saúde (avaliada, a partir do dia da parada até 1 ano depois), a saber: a avaliação do grau de dependência da nicotina, a presença de outros fumantes no convívio da pessoa, a fase de motivação em relação à mudança e o número de tentativas anteriores para parar de fumar ${ }^{26,28}$.

O emprego de medicamentos deve ser ponderado com base nessa avaliação prévia. Durante a gravidez, o uso de medicamentos específicos para ajudar a parar de fumar deve ser prescrito mediante avaliação criteriosa, a fim de proteger a saúde do bebê e da mulher28,29,30.

Aconselhar sobre os benefícios do parar de fumar, os malefícios do consumo do tabaco para a saúde humana, a relevância de valorizar projetos de vida sem tabaco, o apoio em situações difíceis e a construção de alternativas para minimizar os sintomas da síndrome de abstinência promovem maior motivação para a tomada de decisão em parar de fumar ${ }^{26}$.

Negociar metas compartilhadas e factíveis contribuem para a um plano de cuidados que reforça a prontidão da mulher em prol da cessação do tabagismo. Oferecer várias alternativas comportamentais e controle de estímulos no ambiente podem preparar melhor para a nova tentativa de abandonar o consumo do tabaco.

O acompanhamento em serviços de saúde que facilita o parar de fumar pode ser presencial ou a distância (monitoramento telefônico, por exemplo) e garante apoio adicional por meio de estratégias de prevenção de recaídas ${ }^{28,31}$.

Em síntese, investir em abordagens sistematizadas e delineadas em parceria com a própria mulher permite uma ação mais efetiva para o controle do tabagismo e a prevenção de recaídas, visto que a gravidez configura um momento ímpar para a sensibilização sobre a cessação do fumar, considerando-se ainda a importância de que essa parada perdure durante o período da amamentação.

\section{CONCLUSÃO}

Com este estudo, foi possível verificar uma prevalência relativamente baixa (11\%) de tabagismo na gestação, ao considerar o achado encontrado em outros estudos ao longo da década passada. Mas, no processo de transformação do comportamento do fumar não foram observadas mudanças nos fatores relacionados à experimentação precoce do consumo do tabaco, aos motivos para o início do tabagismo e à repercussão no peso do bebê ao nascer.

A respeito do grau de dependência da nicotina das fumantes entrevistadas, a maioria obteve grau leve. Como esperado, foi evidente a relação 
entre o número maior de cigarros consumidos e um grau de dependência grave.

O valor médio do peso dos recém-nascidos de mães não fumantes foi 210 gramas maior que o peso dos recém-nascidos das mães fumantes, concluindo, assim, que a diferença foi estatisticamente significativa. Quando considerado o número de cigarros fumados por dia, os testes não mostraram diferença significativa entre o peso do recém-nascido de mães não fumantes e fumantes e entre as fumantes.

Observou-se a necessidade de intensificar a abordagem sobre o tabagismo no pré-natal, para que se alertem às grávidas dos riscos e malefícios do cigarro, causados tanto à mãe como ao bebê. Há ainda muitas mulheres que desconhecem informações básicas sobre o assunto.

A condição de grávida faz com que as muIheres mudem o comportamento sobre o ato de fumar: muitas param, outras diminuem o número de cigarros fumados e a pequena minoria mantém o tabagismo. É majoritária a vontade do cessar de fumar.

Formar profissionais de saúde que valorizem modelos de aconselhamento sistemáticos e baseados em evidências para a cessação do fumar durante a gravidez pode contribuir sobremaneira para a efetiva proteção da saúde materna e da primeira infância e a construção de projetos de vida incompatíveis com a dependência e a toxicidade do tabaco.

\section{REFERÊNCIAS}

1. World Health Organization (Geneva). WHO report on the global tobacco epidemic, 2011: warning about the dangers of tobacco. World Health Organization, 2011.

2. Ministério da Saúde (Brasil), Instituto Nacional de Câncer José Alencar Gomes da Silva (INCA) [Internet]. Programa Nacional de Controle do Tabagismo. [updated 2012 Sep 15; cited Sep 15] Available from: http://www.inca.gov.br/tabagismo/. Acesso em 26 jan. 2012.

3. Ministério da Saúde (Brasil), Instituto Nacional de Câncer José Alencar Gomes da Silva (INCA). Estimativa 2012: incidência de câncer no Brasil. Rio de Janeiro: Inca, 2011.

4. Zhang L, Chica DAG, Cesar JA, Mendoza-Sassi RA, Beskow B, Larentis N, Blosfeld T. Tabagismo materno durante a gestação e medidas antropométricas do recém-nascido: um estudo de base populacional no extremo sul do Brasil. Cad. Saúde Pública. 2011 Set; 27 (9): 1768-1776.

5. Galão AO, Sodre AS, Gerhardt M, Faertes TH, Krüger MS, Pereira DF, Borba CM. Efeitos do fumo materno durante a gestação e complicações perinatais. Rev HCPA. 2009; 29(3): 218-224.

6. Montenegro CAB, Rezende Filho J. Obstetrícia fundamental. 12. ed. Rio de Janeiro: Guanabara Koogan; 2011.724 p.

7. Possato M, Parada CMGL, Tonete VLP. Representação de gestantes tabagistas sobre o uso do cigarro: estudo realizado em hospital do interior paulista. Rev Esc Enferm USP. 2007; 41(3): 434-440.

8. Machado JB, Lopes MHI. Abordagem do tabagismo na gestação. Scientia Medica. 2009 Abr-Jun; 19(2): 75-80. 2009.

9. Palmeira VFS, Tsunechiro MA. Hábito de fumar em mulheres no período gestacional [CDROM]. In: Simpósio Internacional de Iniciação Científica da Universidade de São Paulo 9., 2001, Ribeirão Preto. Resumos. São Paulo, Universidade de São Paulo, 2001a.

10. Palmeira VFS, Tsunechiro MA. Grau de dependência à nicotina de mulheres no período gestacional [CDROM]. In: Simpósio Internacional de Iniciação Científica da Universidade de São Paulo 9., 2001, Ribeirão Preto. Resumos. São Paulo, Universidade de São Paulo, 2001b.

11. Freire K, Padilha PC, Saunders C. Fatores associados ao uso de álcool e cigarro na gestação. Rev. Bras. Ginecol. Obstet. 2009 Jul; 31(7): 335-341.

12. Reis LG, Silva CJ, Trindade A, Abrahão M, Silva VA. Women who smoke and stop during pregnancy: who are they?. Rev. Bras. Saúde Mater. Infant. 2008 Mar; 8(2): 217-221.

13. Motta, GCP, Echer IC, Lucena AF. Fatores associados ao tabagismo na gestação. Rev. Latino-Am. Enfermagem. 2010 Jul-Ago; 18(4): 809-815.

14. Araújo AJ, Menezes AMB., Dórea AJPS, Torres BS, Viegas CAA, Silva CAR et al. Diretrizes para Cessação do Tabagismo. J. Bras. Pneumol. 2004 Ago; 30(2): S1- S76.

15. Souza EST, Crippa JAS, Pasian SR, Martinez JAB. Escala Razões para Fumar da Universidade de São Paulo: um novo instrumento para avaliar a motivação para fumar. J. Bras. Pneumol.. 2010 Dez; 36(6): 768-778.

16. Rozov T, Fiss E, Catherino P, Perestrelo MI, Nomura M. Hábito de fumar das gestantes e parturientes de um hospital universitário e seus conhecimentos sobre os efeitos do fumo em fetos e lactentes. Arq. Med. ABC. 2004 Jan-Jun; 29(1): $28-36$. 17. D Hammond1, Fong GT, McNeill A, Borland R, Cummings KM. Effectiveness of cigarette warning labels in informing smokers about the risks of smoking: findings from the International Tobacco Control (ITC) Four Country Survey. Tob Control.2006; 15(3): 19-25. 
18. Nakamura MU, Alexandre SM, Santos JFK, Souza E, Sass N, Beck APA et al . Obstetric and perinatal effects of active and/or passive smoking during pregnancy. São Paulo Med. J.,2004 Mai; 122(3): 94-98.

19. Mello PRB, Pinto GR, Botelho C. Influência do tabagismo na fertilidade, gestação e lactação. Jornal de Pediatria. 2001; 77(4): 257-264.

20. Lombardi EMS, Prado GF, Santos UP, Fernandes FLA. O tabagismo e a mulher: riscos, impactos e desafios. J. Bras. Pneumol.,2011 Fev; 37(1): 118-128.

21. Borland T et al. Exploring the adequacy of smoking cessation support for pregnant and postpartum women. BMC Public Health, 2013 [cited 31 aug 2013]; 13(472): 01-11. Available from: http://www.biomedcentral.com/1471-2458/13/472.

22. Flemming K, Graham H, Heirs M, Fox D, Sowden A. Smoking in pregnancy: a systematic review of qualitative research of women commence pregnancy as smokers. Journal of Advanced Nursing. 2013 [cited 30 jul. 2013]; 9(5): 1023-1036.

112 Available from: http://onlinelibrary.wiley.com/doi/10.1111/jan.12066/abstract?deniedAccessCustomis edMessage= \&userlsAuthenticated=false.

23. Ferreira SAL, Teixeira CC, Corrêa APA, Lucena AF, Echer IC. Motivos que contribuem para indivíduos de uma escola de nível superior tornarem-se ou não tabagistas. Rev. Gaúcha Enferm., 2011 Jun; 32(2): 287-293.

24. Instituto Brasileiro de Geografia e Estatística (Brasil). PNAD (Pesquisa Nacional por Amostra de Domicílios). Tabagismo 2008. Rio de Janeiro: IBGE; 2009.

25. Leopércio W, Gigliotti A. Tabagismo e suas peculiaridades durante a gestação: uma revisão. J Bras Pneumol. 2004; 30(2): 176-184.

26. Oliveira AAP, Chiesa AM. Prevenção e controle do tabagismo. In: Harada MJCS, Pedreira MLG, Viana DL, organizadoras. Promoção da Saúde: fundamentos e práticas. São Caetano do Sul: Yendis, 2012.

27. Khouri DG, Santos CD, Tunala RG, Oliveira AAP, Silva ACCG, Ferreira Junior M. Aconselhamento em promoção da saúde. In: Nunes MPT, Lin CA, Martins MA, Augusto KL, Pavanelli MC, Souza MRB et al, organizadores. Clínica médica: grandes temas na prática. São Paulo, Atheneu; 2010.pg 43-52.

28. Fiore MC, Jaén CR, Baker TB, Bailey WC, Benowitz NL, Curry SJ et al. Treating tobacco use and dependence: 2008. Update; 2008.

29. U.S. Preventive Services Task Force. The guide to clinical preventive services 2010-2011: recommendations of the U.S. Preventive Services Task Force; 2010.

30. Reichert J, Araújo AJ, Gonçalves CMC, Godoy I, Chatkin JM, Sales MPU et al. Diretrizes para a cessação do tabagismo - 2008. J Bras Pneumol. 2008; 34(10):845-80.

31. Corrêa EC, Oyama SMR. Aconselhamento telefônico para a cessação do tabagismo. Rev Gaúcha Enferm. 2008;29(4):513-9.

Recebido em: 03 de janeiro de 2014. Aprovado em: 02 de junho de 2015. 